Virginia Commonwealth University

vCU Scholars Compass

1979

\title{
A Comparison of Amphotericin B Alone and Combined with Flucytosine in the Treatment of Cryptoccal Meningitis
}

John E. Bennett, M.D.

National Institutes of Health

William E. Dismukes , M.D.

University of Alabama - Birmingham

Richard J. Duma , M.D.

Virginia Commonwealth University, Medical College of Virginia

See next page for additional authors

Follow this and additional works at: http://scholarscompass.vcu.edu/intmed_pubs

Part of the Medicine and Health Sciences Commons

From The New England Journal of Medicine, Bennett, J.E., Dismukes, W.E., Duma, R.J., et al., A Comparison of Amphotericin B Alone and Combined with Flucytosine in the Treatment of Cryptoccal Meningitis, Vol. 301, Page 126, Copyright (C) 1979 Massachusetts Medical Society. Reprinted with permission.

\section{Downloaded from}

http://scholarscompass.vcu.edu/intmed_pubs/61

This Article is brought to you for free and open access by the Dept. of Internal Medicine at VCU Scholars Compass. It has been accepted for inclusion in Internal Medicine Publications by an authorized administrator of VCU Scholars Compass. For more information, please contact libcompass@vcu.edu. 


\section{Authors}

John E. Bennett , M.D.; William E. Dismukes , M.D.; Richard J. Duma , M.D.; Gerald Medoff, M.D.; Merle A. Sande , M.D.; Harry Gallis , M.D.; John Leonard , M.D.; Branch T. Fields , M.D.; Major Bradshaw , M.D.; Hubert Haywood, M.D.; Zell A. McGee , M.D.; Thomas R. Cate , M.D.; C. Glenn Cobbs , M.D.; John F. Warner, M.D.; and David W. Alling, M.D., Ph.D. 
15. Ginsberg CM, McCracken GH Jr, Rae S, et al: Haemophilus influenzae type b disease: incidence in a day-care center. JAMA 238:604-607, 1977

16. Melish ME, Nelson AJ, Martin. TE, et al: Epidemic spread of $\mathbf{H}$. influenzae type b (HIB) in a day care center. Pediatr Res 10:348, 1976

17. Ward Jl, Gorman G, Phillips C, et al: Hemophilus influenzae type b disease in a day-care center: report of an outbreak. J Pediatr 92:713-717, 1978

18. Granoff D, Gilsdorf J, Gessert C, et al: Haemophilus influenzae type B disease in a day-care center: eradication of carrier state by rifampin. Pediatrics 63:397-401, 1979

19. Wycliffe N, Landwirth J: Haemophilus influenzae Type b infections. Am J Dis Child 132:431, 1978

20. Glode MP, Schiffer MS, Robbins JB, et al: An outbreak of Haemophilus influenzae type b meningitis in an enclosed hospital population. J Pediatr 88:36-40, 1976

21. Filice GA, Andrews JS Jr, Hudgins MP, et al: Spread of Haemophilus influenzae: secondary illness in household contacts of patients with $H$ influenzae meningitis. Am J Dis Child 132:757-759, 1978
22. Anderson P: Intrinsic tritium labeling of the capsular polysaccharide antigen of Haemophilus influenzae type b. J Immunol 120:866-870, 1978

23. Documenta Geigy: Scientific tables. Sixth edition. Edited by K Diem. Ardsley, New York, Geigy Pharmaceuticals, 1962, p 151

24. The Meningococcal Disease Surveillance Group: Meningococcal disease: secondary attack rate and chemoprophylaxis in the United States, 1974. JAMA 235:261-265, 1976

25. McCormick JB, Bennett JV: Public health considerations in the management of meningococcal disease. Ann Intern Med 83:883-886, 1975

26. Smith DH, Peter G, Ingram DL, et al: Responses of children immunized with the capsular polysaccharide of Haemophilus influenzae. type b. Pediatrics 52:637-644, 1973

27. Yogev R, Lander HB, Davis AT: Effect of TMP-SMX on nasopharyngeal carriage of ampicillin-sensitive and ampicillin-resistant Hemophilus influenzae type B. J Pediatr 93:394-397, 1978

28. Kirven LA, Thornsberry C: Minimal bactericidal concentration of sulfamethoxazole-trimethoprim for Haemophilus influenzae: correlation with prophylaxis. Presented at the 18th Interscience Conference on An timicrobial Agents and Chemotherapy, Atlanta, October 1-4, 1978

\section{A COMPARISON OF AMPHOTERICIN B ALONE AND COMBINED WITH FLUCYTOSINE IN THE TREATMENT OF CRYPTOCCAL MENINGITIS}

John E. Bennett, M.D., William E. Dismukes, M.D., Richard J. Duma, M.D., Gerald Medoff, M.D., Merle A. Sande, M.D., Harry Gallis, M.D., John Leonard, M.D., Branch T. Fields, M.D., Major Bradshaw, M.D., Hubert Haywood, M.D., Zell A. McGee, M.D., Thomas R. Cate, M.D., C. Glenn Cobbs, M.D., John F. Warner, M.D. and David W. Alling, M.D., Ph.D.

\begin{abstract}
We compared amphotericin B therapy for cryptococcal meningitis with a newer regimen containing both amphotericin B and flucytosine. In 50 patients with 51 courses of therapy adherent to the protocol, 27 courses were with amphotericin B and 24 with the combination. Even though the combination regimen was given for only six weeks and amphotericin B for 10 weeks, the combination cured or improved more patients (16 vs. 11), produced fewer
\end{abstract}

$\mathrm{C}$ RYPTOCOCCAL meningitis was a uniformly fatal disease before the introduction of amphotericin B. ${ }^{1}$ Although cures are obtained in 53 to 58 per cent of patients, ${ }^{2,3}$ amphotericin B therapy requires prolonged treatment and toxicity is substantial. Flucytosine can also cure this infection, but secondary drug resistance and a low proportion of cures make this drug undesirable as a single agent. ${ }^{4}$ In vitro ${ }^{5}$ and in vivo ${ }^{6}$ evidence that amphotericin $B$ and flucytosine were at least additive in their effects against cryptococcosis prompted clinical trials of the combination. In a prospective, though uncontrolled, $\operatorname{trial}^{7}$ of combination therapy in 15 patients with cryptococcal

From the National Institutes of Health, Bethesda, MD, the University of Alabama School of Medicine, Birmingham, AL, the Medical College of Virginia, Richmond, VA, Washington University School of Medicine, St. Louis, MO, the University of Virginia School of Medicine, Charlottesville, VA, Duke University School of Medicine, Durham, NC, Vanderbilt University School of Medicine, Nashville, TN, the Veterans Administration Hospital and University of Arkansas College of Medicine, Little Rock, AR, Baylor University School of Medicine, Houston, TX, and Ohio State University School of Medicine, Columbus, $\mathrm{OH}$ (address reprint requests to Dr. Bennett at the Clinical Center, Bldg. 10, Rm. $11 \mathrm{~N}-210$, National Institutes of Health, Bethesda, MD 20014)

Supported in part by the National Institute of Allergy and Infectious Diseases and by the John A. Hartford Foundation. failures or relapses (three vs. 11), more rapid sterilization of the cerebrospinal fluid $(P<0.001)$ and less nephrotoxicity $(P<0.05)$ than did amphotericin $B$ alone. The number of deaths was the same (five) with each regimen. Adverse reactions to flucytosine occurred in 11 of 34 patients but were not life threatening. We conclude that combined flucytosine-amphoericin B therapy is the regimen of choice in cryptococcal meningitis. (N Engl J Med 301:126-131, 1979)

meningitis, eight patients were cured at eight to 34 months, four died of other causes within 11 months of completion of therapy, and three died of cryptococcosis despite therapy. No patient relapsed. These results were so encouraging that a prospective collaborative study was undertaken to compare six weeks of flucytosine-amphotericin B with 10 weeks of amphotericin $\mathrm{B}$ alone. The regimen of amphotericin $\mathrm{B}$ chosen was similar to that advocated by Drutz. ${ }^{8}$

\section{Materials and Methods}

\section{Admission Criteria}

Patients could be entered into the study if informed consent was obtained and one of two criteria was met: (1) positive cerebrospinal-fluid india-ink smear or culture, or (2) cerebrospinal-fluid and clinical findings compatible with cryptococcal meningitis plus one of the following: positive culture from another site, positive latex-agglutination test for cryptococcal antigen in the spinal fluid or biopsy evidence of intracranial cryptococcosis. Patients were kept in the study only if Cryptococcus neoformans was documented in cultures of cerebrospinal fluid, brain or meninges.

No patient was excluded because of age, clinical status or underlying disease. Partially treated patients were excluded if they had received more than $2 \mathrm{mg}$ of amphotericin B per kilogram of body weight or $1 \mathrm{~g}$ of flucytosine per kilogram. Patients in relapse 
were admissible if they had received no therapy for at least one month and had a positive cerebrospinal-fluid culture after the last dose of systemic antifungal therapy; these patients were randomized to either regimen irrespective of the drugs used in the first course of therapy.

\section{Drug Regimens}

A 10-week regimen of amphotericin B alone $(0.4 \mathrm{mg}$ per kilogram of body weight per day for 42 days, followed by $0.8 \mathrm{mg}$ per kilogram every other day for 28 days) was compared with a six-week regimen of amphotericin B (0.3 mg per kilogram per day) and flucytosine (150 mg per kilogram per day divided in six hourly doses). Before the study, the protocol regimens and permissible limits were defined. On both regimens, the dosage of amphotericin B was not specified for the first three days to permit gradual dose increments when necessary. Dosages of flucytosine below those shown in Table 1 were permitted in patients with azotemia according to a published nomogram. 9 A patient was regarded as adhering to the protocol if therapy was within all the limits shown in Table 1 . After the study had begun, the permissible limits of duration and drug days were considered unrealistically narrow and therefore were expanded, producing the limits shown in Table 1. With the original limits, therapy in two additional patients would not have conformed with the protocol because of error. On another occasion, it was decided that the protocol did not permit adequate dose reduction of flucytosine in patients with toxicity. The lowest permissible limit for flucytosine was then reduced from two thirds to half the usual dose, and the lowest permissible total dose was reduced to $3150 \mathrm{mg}$ per kilogram. Because of this change one additional patient was included, whose therapy would not otherwise have conformed with the protocol owing to toxicity.

Up to three doses of intrathecal amphotericin B were permitted in either regimen, but only two patients received such treatment during the protocol regimen. One patient was given a single dose, and another received 18 doses and thus did not adhere to the protocol. No medical therapy for cryptococcosis other than that described in the protocol was permitted.

\section{Randomization of Patients and Storage of Data}

Each patient was randomized by having the investigator telephone a central facility that kept a balanced randomization sequence in sealed envelopes. Results of treatment were assessed by a predetermined series of laboratory tests and recorded on standardized data sheets. At a central facility, data were transferred to a computer for subsequent retrieval and analysis. Among the laboratory data required by the study were a biweekly complete blood count, including platelet count, and weekly tests of liver and renal function. Lumbar puncture was required weekly for six weeks in all patients and biweekly thereafter in patients receiving the 10 -week course.

Table 1. Drug Regimens.

\begin{tabular}{lcc}
\hline \hline Treatment Regimen & Amphotericin B & $\begin{array}{c}\text { AMphotericin B- } \\
\text { Flucytosine }\end{array}$ \\
Duration (days) & 70 & 42 \\
Dosage (mg/kg/day) & & \\
$\quad$ Amphotericin B & 0.4 & 0.3 \\
Flucytosine & 0 & 150 \\
Permissible limits & & \\
Duration (days) & $60-82$ & $36-48$ \\
Drug days & $52-62$ & $36-48$ \\
Low-dose days* & $\leqslant 17$ & $\leqslant 10$ \\
High-dose days $\dagger$ & $\leqslant 10$ & $\leqslant 10$ \\
Total dose (mg/kg) & & \\
Amphotericin B & $21-35$ & $9.4-15.7$ \\
Flucytosine & 0 & $3150-7820$
\end{tabular}

*No. of days on which the patient received $<2 / 3$ the specified dose of amphotericin $B$ or $<1 / 2$ the specified dose of flucytosine.

+ No. of days on which the patient received $>11 / 3$ the specified dose of either flucytosine or amphotericin B.
Table 2. Results in Patients Whose Course Adhered to the Protocol and in Those Whose Course Did Not.

\begin{tabular}{|c|c|c|c|c|}
\hline \multirow[t]{3}{*}{ Status* } & \multicolumn{2}{|c|}{ ADHERENT } & \multicolumn{2}{|c|}{ INADHERENT } \\
\hline & $\begin{array}{l}\text { AMPHO- } \\
\text { TERICIN B }\end{array}$ & $\begin{array}{l}\text { AMPHO- } \\
\text { TERICIN B- } \\
\text { FLUCYTO- } \\
\text { SINE }\end{array}$ & $\begin{array}{l}\text { AMPHO- } \\
\text { TERICIN B }\end{array}$ & $\begin{array}{l}\text { AMPHO- } \\
\text { TERICIN B- } \\
\text { FLUCYTO- } \\
\text { SINE }\end{array}$ \\
\hline & \multicolumn{4}{|c|}{ no. of patients } \\
\hline Cured & 7 & 13 & 3 & 2 \\
\hline Improved & 4 & 3 & 1 & 5 \\
\hline Relapsed & 5 & 1 & 0 & 0 \\
\hline Failed full course & 6 & 2 & 0 & 0 \\
\hline Died during therapy & 5 & 5 & 1 & 3 \\
\hline $\begin{array}{l}\text { Proportion cured or } \\
\text { improved }\end{array}$ & $\overline{11 / 27}$ & $\overline{16 / 24}$ & $\overline{4 / 5}$ & $\overline{7 / 10}$ \\
\hline
\end{tabular}

*Patients considered cured had no evidence of active cryptococcosis on examinations including lumbar puncture at least $1 \mathrm{yr}$ after therapy. Those considered improved were discharged with negative cultures for $C$ neoformans \& had no evidence of active cryptococcosis when last seen, but either the last examination was performed less than 1 yr after therapy, examination $1 \mathrm{yr}$ after therapy was incomplete or patient died of other causes within $1 \mathrm{yr}$ after therapy. Those defined as relapsed were discharged as improved but, on readmission, $C$ neoformans was isolated from cerebrospinal fluid or urine. Those who failed full course had positive cultures during last 2 wk of therapy or were retreated at end of course for presumed therapeutic inadequacy. Those who died during therapy died of any cause during same admission that protocol therapy was given.

\section{Prospective Design}

The central question was whether combination therapy for a shorter period would be at least as good as conventional therapy. For statistical purposes, the hypothesis was that combination therapy would result in 20 per cent fewer favorable responses than would conventional therapy. Twenty per cent was chosen arbitrarily as the amount of difference with substantial clinical importance. We hoped to reject the above hypothesis at the 5 per cent level of significance and accept the alternative - that the two regimens were equally effective. The group felt that the shorter duration and lower total dose of amphotericin B in the combination regimen would make this therapy attractive even if it proved to be only "as good as" conventional therapy. For the purposes of this study, both improvement and cure were treated as favorable responses (therapeutic responses are defined in Table 2).

\section{Results}

\section{Study Population}

The study was stopped after 24 months, in February, 1976, because the goal of the study had apparently been achieved. In addition, some of the investigators believed that the effects of the conventional regimen were inadequate. During the 24 months of the study, 78 patients were entered -25 at the University of Alabama, 12 at the Medical College of Virginia, 10 at the National Institutes of Health, seven at the University of Virginia, seven at Washington University, seven at Duke University, four at Vanderbilt University, three at the University of Arkansas, two at Baylor University, and one at Ohio State University. By chance, 39 patients were assigned to each regimen. To determine the validity of the randomization process, we compared the two groups in terms of age, sex, race, underlying disease, the presence of antigen in cerebrospinal fluid and serum and of $C$. neoformans in cultures from extraneural sites. No important differences were found between the groups. 
There was a considerable difference between institutions in the proportion of patients in the study who had serious underlying disease. This variability was anticipated and considered to be one of the strengths of a multicenter trial. The use of combined results from quite different institutions was believed to broaden their applicability.

Among the 78 patients, nine did not meet the criteria for remaining in the study. Three had cryptococcosis in other organs but no meningitis. Four had chronic meningitis without laboratory evidence of cryptococcosis. Two others had cryptococcal meningitis as judged by clinical course, cryptococcal antigen in cerebrospinal fluid and subsequent cure by amphotericin B, but the disease was not confirmed by culture. Three other patients were eligible for retention in the study but provided insufficient information to be included in subsequent tabulations; one died untreated, and two signed out during the first four days of treatment. The characteristics of the remaining 66 patients appear in Table 3. Three patients who relapsed after amphotericin $B$ were entered into the study and were among the 66; one had been treated with amphotericin B under this protocol and was rerandomized, by chance, to the group receiving combination therapy.

\section{Adherence to Protocol}

Among the 66 evaluable courses of therapy, 51 adhered to the protocol. Fifteen were judged not to have adhered -10 to the combination therapy and five to amphotericin B alone. The two major factors causing inadherence were drug toxicity and inability of the investigator, when acting as a consultant, to control the patient's management. Drug toxicity sufficient to cause inadherence was encountered in seven patients and in each case was ascribed solely to flucytosine by the individual investigators. Toxicity from flucytosine usually led to its premature discontinuation. Amphotericin B was then continued, usually in

Table 3. Characteristics of the Study Group.

\begin{tabular}{lcc}
\hline \hline FEATURE & $\begin{array}{c}\text { AMPHO- } \\
\text { TERICIN B }\end{array}$ & $\begin{array}{c}\text { AMPHO- } \\
\text { TERICIN B- } \\
\text { FLLCYTO- } \\
\text { SINE }\end{array}$ \\
No. of patients & 32 & 34 \\
Age range (yr) & $17-82$ & $18-73$ \\
Mean age (yr) & 43 & 48 \\
Cerebrospinal-fluid india ink & 62 & 59 \\
(\% positive) & 28 & 35 \\
Positive extraneural culture (\%) & 2 & 1 \\
Relapse from prior therapy & & \\
Underlying disease (no. of cases) & 2 & 5 \\
$\quad$ Chronic lymphocytic leukemia & 4 & 3 \\
$\quad$ Lymphoma & 8 & 5 \\
$\quad$ Renal transplant recipient & 5 & 3 \\
$\quad$ Chronic hepatitis or cirrhosis & 3 & 5 \\
$\quad$ Diabetes mellitus & 2 & 0 \\
$\quad$ Sarcoidosis & & \\
$\quad$ Total with underlying & 24 & 21 \\
$\quad$ disease & & \\
\hline
\end{tabular}

increased doses. Once the course no longer adhered to the protocol, the dose and duration of therapy were no longer specified.

\section{Therapeutic Efficacy (Table 2)}

The final results were sufficient to meet the study's objective in that the hypothesis that we hoped to reject could be rejected at the 1 per cent level of significance. Clearly, the combination was not 20 per cent worse than conventional therapy. Rather, the results favored the combination. In the total group of 66 patients, 23 of 34 ( 68 per cent) were cured or improved by the combination, and 15 of 32 (47 per cent) by amphotericin B alone $\left(\chi^{2}=2.12 ; \mathrm{P}>0.05\right)$. Results were quite similar when courses not adhering to protocol were excluded totally; 16 of 24 patients (67 per cent) were cured or improved by the combination, and 11 of 27 (41 per cent) by amphotericin B alone $\left(\chi^{2}=2.47\right.$; $\mathrm{P}>0.05)$. When failure to adhere was counted as an unfavorable outcome of therapy, of 34 patients 16 (47 per cent) were cured or improved by the combination vs. 11 of 32 ( 34 per cent) by amphotericin $B$ alone $\left(\chi^{2}=0.67 ; \mathrm{P}>0.10\right)$.

Additional findings must be considered. In the first place, all six patients listed under "failed full course" of amphotericin B in Table 2 failed to convert their cerebrospinal-fluid cultures during the protocol regimen. All died with active cryptococcosis despite further therapy. Among the five who relapsed during treatment with amphotericin $B$, three died with active cryptococcosis during retreatment. In contrast to these eleven patients, the three whose courses adhered to combination therapy but who relapsed or failed were all eventually discharged with no evidence of active cryptococcosis. In two patients who failed a full course of combination therapy, all cultures converted to negative, but the cerebrospinal fluid remained abnormal. After 23 and 87 days of post-treatment observation, these patients were retreated because this abnormality persisted, although failure of therapy had not been documented. In all, there were 15 deaths among 32 patients treated with amphotericin $\mathrm{B}$ alone (47 per cent), as compared to only eight deaths among the 34 patients receiving the combination (24 per cent) $\left(\chi^{2}=3.96\right.$; $\left.\mathrm{P}<0.05\right)$. When results of retreatment are included, 19 of 24 patients whose courses adhered to combination therapy ( 79 per cent) and only 13 of 27 who received amphotericin B were eventually discharged with inactive cryptococcosis $\left(\chi^{2}=3.99 ; \mathrm{P}<0.05\right)$. When courses that did not adhere are included, the final results of all courses of therapy still favored the combination ( 76 per cent cured or improved) over amphotericin B alone (53 per cent). Another factor suggesting the combination was superior was the observation that cerebrospinal-fluid culture converted from positive to negative more rapidly in patients given combination therapy (Fig. 1) $(\mathrm{P}<0.001$, Wilcoxon two-sample test $)$.

The two regimens did not differ in the rate of improvement in cryptococcal antigen in serum or glu- 


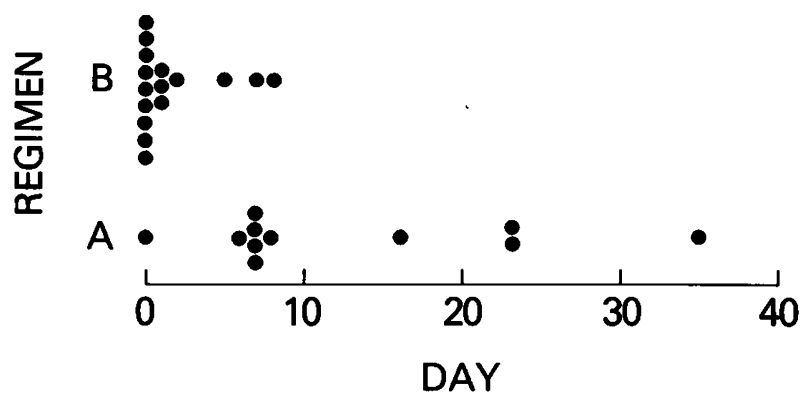

Figure 1. Day of Last Positive Culture among Patients Who Were Cured or Improved and Whose Course Adhered to the Protocol.

Each dot represents one patient. The A denotes amphoteri$\operatorname{cin} B$ alone, and the $B$ combined therapy with amphotericin B and flucytosine.

cose, protein, cell count, cryptococcal antigen or india-ink tests in cerebrospinal fluid.

\section{Immunosuppressive Therapy (Table 4)}

Among 51 patients whose treatment adhered to the protocol, 26 received immunosuppressive therapy during the first week of antifungal therapy. In the 10 receiving azathioprine and prednisone these drugs were given throughout antifungal therapy, whereas in six of 16 patients, the other regimens were given intermittently or were discontinued. None of the differences between amphotericin B and the combination regimen was statistically significant.

\section{Antigen Testing}

Cryptococcal antigen was measured by latex agglutination in cerebrospinal fluid within a week of institution of therapy in 55 of the 66 patients who were retained in the study and within a week of the end of therapy in 40 of these patients. The geometric mean titer was higher in unsuccessfully treated patients than in cured and improved patients, both at the beginning of treatment $(1: 676$ vs. $1: 94 ; \mathrm{P}<0.01)$ and at the end $(1: 137$ vs. $1: 12 ; P<0.02)$. Only two of 55 patients had negative titers on admission. Serum antigen titers in 44 patients at the start of therapy and 28 at the end were generally lower and less helpful than titers in the cerebrospinal fluid. A twofold drop in antigen titers in cerebrospinal fluid or serum during therapy did not distinguish success from failure, although paired specimens were not analyzed.

\section{Flucytosine Susceptibility}

Isolates from 33 of 34 patients receiving flucytosine were assessed for drug susceptibility.$^{10}$ The one isolate not studied was from a patient who died on the first day of treatment. All isolates had a minimal inhibitory concentration $\leqslant 2.5 \mathrm{mg}$ of flucytosine per liter, which denotes susceptibility. The minimal inhibitory concentration in the patient who relapsed rose from $\leqslant 2.5$ to $>320 \mathrm{mg}$ per liter; this patient was successfully retreated with combination therapy.

\section{Side Effects}

Side effects from flucytosine appeared to be present in 11 of 34 patients randomized to combination therapy and in one of six receiving flucytosine after failure of amphotericin B alone - that is, in 12 of 40 patients ( 30 per cent). As shown in Table 5 , nine patients had leukopenia or thrombocytopenia or both. None of the 12 with side effects was receiving cytotoxic agents, although two had lymphoma (Cases 1 and 11), and one had hypersplenism from cirrhosis (Case 19). Three patients had diarrhea, usually associated with abdominal cramps. Three had an erythematous, diffuse maculopapular rash over most of the body. All reactions began 10 to 26 days after starting flucytosine except the rash in Case 14. Eight patients who received reduced doses of flucytosine because of azotemia nevertheless later had leukopenia, thrombocytopenia or diarrhea.

Mild leukopenia developed in three patients receiving amphotericin $\mathrm{B}$ alone. The means of the two lowest leukocyte counts in these patients were 3.2, 3.8 and $4.2 \times 10^{9}$ per liter $(3200,3800$ and 4200 per cubic millimeter). Amphotericin B was not discontinued because of-these abnormalities.

The two groups did not differ in terms of anemia or hypokalemia, and no abnormalities of serum alkaline phosphatase or transaminase were caused by either regimen. Nephrotoxicity was studied in patients with normal serum creatinine levels on admission and no interfering condition during therapy. The mean of the two highest serum creatinine determinations in 16 patients receiving amphotericin B was $200 \mu \mathrm{mol}$ per liter (2.3 $\mathrm{mg}$ per deciliter) and in 18 patients receiving the combination was $140 \mu \mathrm{mol}$ per liter $(1.5 \mathrm{mg}$ per deciliter). When each patient's mean maximum value was compared to the pretreatment value by paired sample t-test, the two regimens differed at the 5 per cent level of significance.

\section{Serum Flucytosine Concentrations}

Flucytosine concentrations were determined on 134 serum samples from 22 patients. ${ }^{11}$ Nine of these

Table 4. Effect of Immunosuppressive Therapy on Efficacy.

\begin{tabular}{lcccc}
\hline $\begin{array}{c}\text { IMMLNOSLPPRESIVE } \\
\text { THERAPY DLRING } \\
\text { 1ST WK OF } \\
\text { Protocol }\end{array}$ & AMPHOTERICIN B & $\begin{array}{c}\text { AMPHOTERICIN B- } \\
\text { FLUCYTOSINE }\end{array}$ \\
$\begin{array}{c}\text { No. OF } \\
\text { PATIENTS }\end{array}$ & $\begin{array}{c}\text { \% CURED OR } \\
\text { IMPROVED }\end{array}$ & $\begin{array}{c}\text { No. OF } \\
\text { PATIENTS }\end{array}$ & $\begin{array}{c}\text { \% CURED OR } \\
\text { IMPROVED }\end{array}$ \\
$\begin{array}{c}\text { Equivalent of } \\
\text { prednisone }\end{array}$ & 3 & 67 & 5 & 80 \\
$\begin{array}{c}\text { Tumor chemotherapy } \\
\text { 10 mg/day } \\
\text { with or without } \\
\text { prednisone }\end{array}$ & 6 & 33 & 2 & 50 \\
$\begin{array}{c}\text { Azathioprine }+ \\
\text { prednisone }\end{array}$ & 7 & 14 & 3 & 33 \\
\begin{tabular}{c} 
None \\
\hline
\end{tabular} & 11 & 55 & 14 & 71 \\
\hline
\end{tabular}


Table 5. Adverse Reactions to Flucytosine.

\begin{tabular}{|c|c|c|c|c|c|c|c|c|c|c|c|c|}
\hline Case no. & 1 & 2 & 11 & 14 & 16 & 17 & 19 & 30 & 41 & 44 & 47 & 72 \\
\hline $\begin{array}{l}\text { Day of therapy toxicity began } \\
\text { Serum creatinine before } \\
\text { toxicity* }(\mathrm{mg} / \mathrm{dl}) \dagger\end{array}$ & - 14 & 21 & 26 & 60 & 26 & 24 & 10 & 24 & 18 & 17 & 14 & 16 \\
\hline Minimum & 1.2 & 1.2 & 3.1 & 0.9 & 1.6 & 1.1 & 0.4 & 0.9 & 1.2 & 0.8 & 2.0 & 1.1 \\
\hline Maximum & 2.6 & 4.1 & 4.1 & 1.3 & 2.3 & 1.5 & 1.0 & 1.8 & 1.9 & 2.0 & 2.4 & 1.6 \\
\hline \multicolumn{13}{|l|}{$\begin{array}{l}\text { Maximum blood abnormality } \ddagger \S \\
\left(\times 10^{9} / \text { liter }\right)\end{array}$} \\
\hline Leukocyte count & 3.3 & 1.5 & - & - & - & 3.3 & 2.7 & 3.8 & - & 3.5 & 3.3 & 2.0 \\
\hline Platelet count & 18 & 119 & 71 & - & - & - & 50 & - & - & - & - & - \\
\hline Diarrhea & - & - & - & - & + & - & - & - & + & - & - & + \\
\hline Rash & - & - & - & + & - & - & + & - & - & - & - & + \\
\hline
\end{tabular}

"Serum creatinine concentrations are given because azotemia is known to decrease flucytosine excretion. ${ }^{9}$

$\dagger$ To convert $\mathrm{mg} / \mathrm{dl}$ to $\mu \mathrm{mol} /$ liter, multiply by 88.4 .

¥No abnormality is designated by minus sign, \& abnormality by plus sign.

$\S$ To convert blood counts to no. per milliliter, multiply by 1000 .

patients had toxicity from flucytosine. In seven of nine, the assay was performed when the patient was receiving the dosage that apparently caused toxicity. In six of seven, the peak blood level during toxicity consistently exceeded $100 \mathrm{mg}$ per liter (Cases 1, 11, 16, 44, 47 and 72). The exception, Case 30 , had mild, stabile leukopenia not requiring dose reduction. Blood levels of flucytosine often exceeded $100 \mathrm{mg}$ per liter in five of 14 patients without toxicity - indicating that flucytosine blood levels were not the sole determinant of toxicity. The effect of mild azotemia on the blood level was evident. At the usual dosage of $150 \mathrm{mg}$ per kilogram of body weight (range, 125 to 160), the mean blood level in 34 specimens from 12 patients with serum creatinine concentrations less than 120 $\mu \mathrm{mol}$ per liter ( $1.4 \mathrm{mg}$ per deciliter) was $78 \mathrm{mg}$ per liter one to two hours after a dose. Among 15 specimens from five patients receiving $150 \mathrm{mg}$ per kilogram of body weight per day and with serum creatinine concentrations of 120 to $160 \mu \mathrm{mol}$ per liter (1.4 to $1.9 \mathrm{mg}$ per deciliter), the mean blood level after one to two hours was $119 \mathrm{mg}$ per liter $(\mathrm{P}<0.001$ by Student's t-test). Mean blood levels of flucytosine six hours after administration also differed; the mean level was $60 \mathrm{mg}$ per liter if the serum creatinine was less than $120 \mu \mathrm{mol}$ per liter ( $1.4 \mu \mathrm{g}$ per milliliter), and the mean level was $99 \mathrm{mg}$ per liter if the serum creatinine was 120 to $160 \mu \mathrm{mol}$ per liter ( 1.4 to $1.9 \mathrm{mg}$ per deciliter $)(\mathrm{P}<0.001)$.

\section{Discussion}

Flucytosine plus amphotericin B was superior to amphotericin $\mathrm{B}$ alone. The ability to achieve a favorable therapeutic result in only six weeks is important, in view of the costs of hospitalization. Culture conversion was more rapid with the combination an observation that helped to explain why results were as good or better with the combination regimen even though the treatment period was four weeks shorter. As shown in Tables 2 and 4, all the differences in outcome favored the combination. When results of retreatment were also included, patients who received the combination had a more favorable final outcome. Although retreatment was not dictated by protocol, the initial course of therapy may have influenced the final outcome by altering the extent of disease in the patients classified as failed or relapsed. The individual case histories support this concept.

Toxicity was the major problem with combination therapy. In six of 34 patients (18 per cent), toxicity was a major factor in the decision to discontinue flucytosine. None of the toxic reactions caused serious problems-in our patients, although deaths from intestinal perforation and neutropenia-induced sepsis have been reported in other studies. ${ }^{4,12,13}$ Blood levels of flucytosine were measured retrospectively and were not used to adjust dosage in our study. Neutropenia, thrombocytopenia and diarrhea were associated with sustained blood levels in excess of $100 \mathrm{mg}$ per liter. Others have noted the same coincidence, using a cutoff of $125 \mathrm{mg}$ per liter. ${ }^{12}$ Toxicity was difficult to evaluate in some patients with severe underlying diseases, but it was clear. that not all patients with elevated blood levels had sustained toxicity. This discrepancy supports the possibility that toxicity is not due directly to flucytosine but to a metabolite. One metabolite of known toxicity, 5-fluorouracil, was detected in serum from some patients in this study. ${ }^{14}$ Whether prospective assessment of blood levels of flucytosine or 5-fluorouracil will reduce the incidence of toxicity is unknown. Biweekly complete blood counts and reduction of dosage in all patients with azotemia are important, but did not completely prevent toxicity in this study. In five patients, toxicity reached its peak eight to 13 days after an appropriate reduction in dosage.

This study raised but did not answer the question of whether the dosage of amphotericin B was too low in the single-drug regimen. The cure rate that we encountered with amphotericin B cannot be compared easily with earlier reports because the characteristics of different patient populations affect outcome. ${ }^{15}$ Still, the proportion of patients cured or improved in this series ( 41 per cent of 27 who adhered to the protocol) is not far below the proportion (53 per cent of 30 patients) cured or improved with the first course of amphotericin B at the National Institutes of Health. ${ }^{2}$ Most of the patients in that series received 0.5 to 0.6 
mg of the drug per kilogram of body weight per day. Another unanswered question is whether intrathecal amphotericin B might have prevented the deaths that occurred in 14 of 66 patients ( 21 per cent) during initial treatment. We did not use intrathecal treatment because we did not think that it would prevent early death. The only patient who received more than a single dose of intrathecal amphotericin B died despite this maneuver.

In view of the equivalent or superior efficacy of flucytosine-amphotericin B therapy, the more rapid sterilization of the cerebrospinal fluid, the reduced duration of treatment and the likely reduction in cost of hospitalization, we have concluded that it is the regimen of choice in cryptococcal meningitis.

We are indebted to Dr. Leo Kaufman of the Center for Disease Control, for performing latex-agglutination tests for cryptococcal antigen, and to Dr. Temple Williams, for assisting in the centralized randomization operation.

\section{REFERENCES}

1. Butler WT, Alling DW, Spickard A, et al: Diagnostic and prognostic value of clinical and laboratory findings in cryptococcal meningitis: a follow-up study of forty patients. N Engl J Med 270:59-67, 1964

2. Spickard A, Butler WT, Andriole V, et al: The improved prognosis of cryptococcal meningitis with amphotericin B therapy. Ann Intern Med $58: 66-83,1963$
3. Sarosi G, Parker JD, Doto IL, et al: Amphotericin B in cryptococcal meningitis: long-term results of treatment. Ann Intern Med 71:1079. 1087,1969

4. Bennett JE: Flucytosine. Ann Intern Med 86:319-322, 1977

5. Medoff G, Comfort M, Kobayashi GS: Synergistic action of amphotericin B and 5-fluorocytosine against yeast-like organisms. Proc Soc Exp Biol Med 138:571-574, 1971

6. Block ER, Bennett JE: The combined effect of 5-fluorocytosine and amphotericin B in the therapy of murine cryptococcosis. Proc Soc Exp Biol Med 142:476-480, 1973

7. Utz JP, Garriques IL, Sande MA, et al: Therapy of cryptococcosis with a combination of flucytosine and amphotericin B. J Infect Dis 132:368373,1975

8. Drutz DJ, Spickard A, Rogers DE, et al: Treatment of disseminated mycotic infections: a new approach to amphotericin B therapy. Am J Med 45:405-418, 1968

9. Schönebeck J, Polak A, Fernex M, et al: Pharmacokinetic studies on the oral antimycotic agent 5-fluorocytosine in individuals with normal and impaired kidney function. Chemotherapy 18:321-336, 1973

10. Block ER, Jennings AE, Bennett JE: Variables influencing susceptibility testing of Cryptococcus neoformans to 5-fluorocytosine. Antimicrob Agents Chemother 4:392-395, 1973

11. Block ER, Bennett JE: Pharmacological studies with 5-fluorocytosine. Antimicrob Agents Chemother 1:476-482, 1972

12. Kauffman CA, Frame PT: Bone marrow toxicity associated with 5fluorocytosine therapy. Antimicrob Agents Chemother 11:244-247, 1977

13. Bryan CS, McFarland JA: Cryptococcal meningitis: fatal marrow aplasia from combined therapy. JAMA 239:1068-1069, 1978

14. Diasio RB, Lakings DE, Bennett JE: Evidence for conversion of 5 fluorocytosine to 5-fluorouracil in humans: possible factor in 5-fluorocytosine clinical toxicity. Antimicrob Agents Chemother 14:903908,1978

15. Diamond RD, Bennett JE: Prognostic factors in cryptococcal meningitis: a study in 111 cases. Ann Intern Med 80:176-181, 1974

\title{
SPECIAL ARTICLE
}

\section{MEDICAL WRITING: ANOTHER LOOK}

\author{
SAUL S. Radovsky, M.D.
}

\begin{abstract}
A LOOK at medical journals shows that good science and good writing rarely go together, and that easily understood writing is often too much to expect. Complaints and critical analyses seem to have had little effect. Thirty-five years ago Alan Gregg said, "The common level of medical and scientific writing in our professional books and journals constitutes the most serious internal limitation to medical education and research."' And four years ago another astute critic, Michael Crichton, wrote, "With medical writing as forbidding as it is...it is impossible to guess the cost...in wasted time, duplicated findings, and buried pearls. But such a cost surely exists, and must be reckoned with."2 Crichton tries to find a reason. Rejecting the arguments that scientists are illiterate, science too complex or editors too demanding of compression, he charges that medical writers write badly on purpose. They would rather impress than inform, he says, and they cast their jargon into weak and obscure constructions to that end.
\end{abstract}

Address reprint requests to 214 N. Main St., Natick, MA 01760.
Although there is some truth to this charge, I think that cultural factors, medical jargon, science itself and imitative writing also contribute.

\section{Culture}

It is traditional to observe that British writers as a group write better than Americans, that their writing is simpler and clearer, more idiomatic and direct, that their overall grasp of language is better than ours. Moreover, ours seems to have been better 75 years ago than it is now. Most native, small-town Americans in their 80's speak simple and accurate English, its idiom as sure as can be.

The 19th century's prudery, with its increasing detachment of language from thought and feeling and its cultured circumlocutions, has to be partly to blame. On its account Captain Marryat could write of having seen little skirts on the legs of American pianos, and Richard Dana could write better prose at 20 than at 44. Its effects went deeper and lasted longer in America than elsewhere, and linger even now. 